\title{
Factores psicosociales de riesgo de consumo de drogas ilícitas en una muestra de estudiantes mexicanos de educación media
}

\author{
Bruno Díaz Negrete ${ }^{1}$ y Raúl García-Aurrecoechea ${ }^{1}$
}

Forma de citar Díaz Negrete B, García-Aurrecoechea R. Factores psicosociales de riesgo de consumo de drogas ilícitas en una muestra de estudiantes mexicanos de educación media. Rev Panam Salud Publica. 2008;24(4): $223-32$.

RESUMEN Objetivos. Identificar factores psicosociales de riesgo de consumo de sustancias ilícitas en estudiantes mexicanos y ofrecer elementos para el diseño de programas de prevención.

Métodos. Estudio transversal no experimental con una muestra de 516 estudiantes de secundaria y bachillerato de seis de las principales ciudades de México. Entre abril y junio de 2005 se aplicó una adaptación de la versión abreviada del Inventario de Tamizaje sobre el Consumo de Drogas (DUSI-R). El análisis comprendió ocho factores: abuso en el consumo de bebidas alcohólicas o drogas, malestar afectivo, bajo control conductual, baja adherencia escolar, baja competencia social, relaciones familiares disfuncionales, aislamiento social y pertenencia a redes sociales desviantes (cuyos integrantes consumen drogas y adoptan actitudes antisociales). Se identificaron factores de predicción del consumo de drogas ilícitas mediante regresión logística y se elaboró un modelo de ecuaciones estructurales para determinar las relaciones entre los factores.

Resultados. Los factores de predicción del consumo de sustancias ilícitas fueron presentar un bajo control conductual con tendencia a actuar impulsivamente y con agresividad, estar vinculado con pares desviantes y estar expuesto con frecuencia a situaciones familiares de conflicto y violencia y al consumo de sustancias ilícitas y alcohol en el hogar. El modelo de ecuaciones estructurales indicó que el consumo de estas sustancias forma parte de un grupo de trastornos de ajuste conductual, determinado directamente por la vinculación con pares desviantes y por una mayor prevalencia de trastornos socioafectivos, e indirectamente por las relaciones familiares disfuncionales.

Conclusiones. Se confirmaron algunas implicaciones de los modelos teóricos propuestos para explicar el consumo de drogas. Estos elementos empíricamente sustentados pueden contribuir al diseño de programas preventivos, principalmente de tipos selectivo e indicado.

Palabras clave Drogas ilícitas, factores de riesgo, estudiantes, México.

1 Centros de Integración Juvenil, A.C., México, D.F., México. La correspondencia se debe dirigir a Bruno Díaz Negrete, Subdirección de Investigación, Centros de Integración Juvenil, A.C., Tlaxcala No. 208, Col. Hipódromo-Condesa, México, D.F. 06100, México. Correo electrónico: investigacion@cij.gob.mx
El consumo de drogas ilícitas en México no solo muestra una tendencia a aumentar (1-3), sino también una complejidad creciente, caracterizada por la expansión del consumo de sustancias como las metanfetaminas y la cocaína de base libre, la existencia de arraigados problemas regionales —como el consumo de heroína en el norte del país-y la combinación de un extenso consumo de cocaína, marihuana y sustancias inhalables con problemas emergentes como el consumo de la droga sintética éxtasis $(4,5)$. Esto plantea la necesidad de desarrollar estrategias de prevención y trata- 
miento orientadas adecuadamente, a fin de garantizar una mayor eficacia y un mejor aprovechamiento de los recursos.

En el campo de la prevención se ha señalado la conveniencia de contar con estrategias de intervención diferenciadas según la población a la que se dirijan, ya sea general (prevención universal), de alto riesgo (prevención selectiva) o a usuarios experimentales y grupos relacionados (prevención indicada) (6-8). En particular, los programas de prevención selectiva e indicada requieren una sólida base teórica y empírica para identificar los diversos factores asociados con el consumo, sobre los cuales se podría incidir con acciones de mayor atingencia y eficacia.

Según diversos estudios, el consumo de sustancias ilícitas es un problema complejo, vinculado con factores de muy diversas índoles que se pueden incorporar a modelos multifactoriales más o menos abarcadores $(7,9)$. Así, algunos autores (10-13) distinguen diferentes tipos de factores, entre ellos: legales y normativos (tolerancia social y falta de observancia de normas y leyes), de disponibilidad de sustancias, de carencias sociales y comunitarias (pobreza, alta densidad y movilidad demográficas, criminalidad, violencia social, alienación y deterioro de vínculos comunitarios), familiares y de ajuste escolar (bajo desempeño y fracaso escolar, y baja motivación por el estudio) y otros factores vinculados con los consumidores de esas sustancias. Igualmente señalan actitudes y conductas desviantes (que se apartan de la norma social convencional, como el consumo de drogas, las actitudes antisociales y el aislamiento social), trastornos de ajuste conductual y social (agresividad, hiperactividad, déficit de atención, aislamiento social y rechazo de los pares) y otros factores de actitud, personalidad y fisiológicos.

Algunos autores como Newcomb (14) reconocen la incidencia de factores socioculturales, interpersonales, psicoconductuales y biogenéticos, mientras otros mencionan el efecto de factores demográficos, socioambientales, conductuales e individuales (15), agrupados según su carácter en socio- comunitarios, familiares, escolares, psicosociales e individuales (8), o bien de factores relacionados con condiciones de malestar que predisponen a la "normalización" del consumo de sustancias ilícitas y con su consumo en sí (alta accesibilidad, expectativas positivas, participación en contextos de consumo, contacto temprano con las drogas, entre otras) (16).

Entre los factores individuales identificados se encuentran el insuficiente control conductual, la rebeldía, las conductas antisociales, la baja adherencia a las normas convencionales y las actitudes favorables al consumo, así como las estrategias de afrontamiento poco efectivas, la escasa habilidad comunicativa, la tendencia a la búsqueda de nuevas sensaciones y experiencias, el malestar afectivo (principalmente, el afecto deprimido), la apatía y la baja autoestima, entre otros.

En el ámbito familiar destacan el consumo de sustancias ilícitas en el seno de la familia, la permisividad, las prácticas inadecuadas de funcionamiento familiar, y la falta, la rigidez o la inconsistencia de las pautas normativas y disciplinarias, así como las relaciones distantes y frías, el rechazo o la hostilidad o, al contrario, el aglutinamiento y la falta de diferenciación entre los miembros de la familia. Asimismo, influyen las relaciones conflictivas, el involucramiento excesivo de los padres, los límites rígidos o difusos, la triangulación del conflicto, las alianzas intergeneracionales y la parentalización de los hijos, es decir la adjudicación a ellos de más responsabilidades familiares y domésticas de las debidas (17).

En el caso de México se ha documentado la relación entre el consumo de drogas ilícitas por parte de los jóvenes y algunos factores, como ser varón y tener una baja percepción del riesgo de consumir drogas (18-20). También se ha señalado que la probabilidad de consumir estas sustancias es mayor cuando hay antecedentes de su consumo, actitudes de tolerancia y permisividad en la familia o entre los pares, y cuando son mayores el acceso a las drogas y las oportunidades de consumo (18-23).
En cuanto al dominio familiar, se ha encontrado que el consumo de sustancias ilícitas está asociado significativamente con la desorganización y la falta de apoyo y control familiar, la separación de los padres, la pérdida del sentido de pertenencia (23), la baja cohesión y la rigidez adaptativa (20), así como con el involucramiento o el distanciamiento excesivos de los padres. En cuanto al contexto escolar, se han identificado como factores de predisposición la baja adherencia escolar, el poco interés por el estudio (23), el bajo desempeño académico y la separación del sistema escolar (19). ${ }^{2}$

En lo que respecta a los factores individuales, se ha documentado que la baja autoestima, la insuficiente asertividad, la incapacidad de controlar los impulsos, las actitudes antisociales y las estrategias de afrontamiento ineficaces implican un mayor riesgo de consumir sustancias ilícitas $(20,24)$. De igual modo, el consumo de drogas parece estar vinculado con los trastornos y los síntomas depresivos y con la exposición temprana o reciente a pérdidas cercanas y eventos traumáticos $(20,23)$, así como con el inconformismo y la insatisfacción con la calidad de vida $(23,25)$. Finalmente, también se ha relacionado el consumo de estas sustancias con el hecho de trabajar en la calle y de disponer de dinero o de un salario $(19,23)$.

Estudios realizados con el Inventario de Tamizaje sobre el Consumo de Drogas (Drug Use Screening Inventory, DUSI) han demostrado que existe una relación significativa entre el consumo de drogas, por una parte, y los trastornos de conducta (26-29), los problemas afectivos y algunos síntomas psicopatológicos, por la otra (26-28). En los hombres que consumen sustancias ilícitas se han observado trastornos de mayor intensidad que en las mujeres,

\footnotetext{
2 Guerra I, Arellánez J, Díaz B. Adherencia escolar y consumo de drogas. México, D.F.: Centros de Integración Juvenil; 1999. (Informe de Investigación 99-07); González D, García R, Córdova A. Uso de sustancias entre adolescentes y su relación con síntomas de depresión y su percepción de sus relaciones familiares. México, D.F.: Centros de Integración Juvenil; 2004. (Informe de Investigación 04-03).
} 
así como un predominio de trastornos de ajuste conductual y laboral, un deficiente desempeño escolar y relaciones con otras personas consumidoras de este tipo de sustancias (30-32). En cambio, en las mujeres predominan los trastornos afectivos, aunque también se han identificado problemas de salud, baja competencia social, deficiente desempeño escolar y vinculación con pares desviantes $(31,32)$.

Estudios realizados con el DUSI en poblaciones latinoamericanas indican que el consumo de drogas ilícitas está relacionado principalmente con alteraciones de la conducta, problemas familiares y de ajuste escolar o laboral, el establecimiento de relaciones con amigos desviantes y que consumen drogas, y con un inadecuado uso del tiempo libre (33-37). Similares hallazgos se han obtenido en estudios realizados en la población mexicana, en los que se han identificado una mayor asociación entre el consumo de drogas, por una parte, y un bajo control conductual, la pertenencia a redes sociales disfuncionales, una baja adherencia escolar y, en menor proporción, un inadecuado uso del tiempo libre, por la otra (38). ${ }^{3} \mathrm{Al}$ igual que en otras latitudes, estos y otros estudios realizados en adolescentes mexicanos han demostrado la validez y confiabilidad del DUSI (39).

Con estos antecedentes, el presente estudio se propuso identificar, a partir de las variables consideradas en el DUSI, factores psicosociales predictores del consumo de sustancias ilícitas en estudiantes mexicanos de educación media y ofrecer elementos para el diseño y aplicación de programas específicos de prevención selectiva e indicada.

\footnotetext{
3 Rodríguez S, Arellánez J, Díaz B, González D. Ajuste psicosocial y consumo de drogas. México, D.F.: Centros de Integración Juvenil; 1999. (Informe de investigación 97-27). Matsuí O, Sánchez M. Uso de sustancias psicoactivas en estudiantes de educación media del estado de Jalisco. Guadalajara: Universidad de Guadalajara, Secretaría de Educación de Jalisco; 2000. Guerrero A, Romero S. Trastornos de ajuste psicosocial entre usuarios de drogas que concluyeron tratamiento en Centros de Integración Juvenil. México, D.F.: Centros de Integración Juvenil; 2001. (Informe de investigación 00-10).
}

\section{MATERIALES Y MÉTODO}

\section{Población de estudio}

Se realizó un estudio transversal no experimental (ex post facto) con datos extraídos de una encuesta administrada originalmente a una muestra no probabilística de 1460 estudiantes de tercer grado de educación secundaria y bachillerato, captados en escuelas de zonas de alto riesgo de seis de las mayores ciudades de México: Ciudad de México, Distrito Federal; Guadalajara, Jalisco; León, Guanajuato; Monterrey, Nuevo León; Puebla, Puebla; y Tijuana, Baja California. Se eligió este grupo de estudio por su elevado riesgo, ya que el consumo de drogas comienza en México alrededor de los 17 años de edad (5); por tener tasas de prevalencia más altas que las observadas en la población general (3); y por su condición de población escolarizada, que además de ofrecer las condiciones propicias para aplicar programas preventivos, constituye un importante grupo diana de varios de los principales programas de atención preventiva en marcha en el país.

Para la presente investigación se emplearon los datos de los 254 estudiantes (49,2\% de la muestra final) que respondieron en la encuesta que habían consumido drogas ilícitas alguna vez en su vida. Los restantes 262 participantes $(50,8 \%$ de la muestra) se seleccionaron de forma aleatoria entre los estudiantes encuestados que manifestaron no haber consumido este tipo de sustancias. De esta forma se contó con dos grupos o muestras independientes, necesarios para realizar los análisis propuestos. El tamaño total de la muestra (516) resultó suficiente para el número de parámetros y relaciones que se utilizarían en la modelación estructural de los datos.

\section{Encuesta}

La encuesta se aplicó en grupos entre abril y junio de 2005 con el consentimiento de las autoridades escolares; se garantizó la confidencialidad de los datos.
Se empleó una adaptación de la versión abreviada del DUSI (DUSI-R), que evalúa trastornos de ajuste psicosocial con respecto a los cuales existe evidencia empírica de una relación significativa con el consumo de drogas y que pueden modificarse mediante intervenciones de prevención o tratamiento (39). El DUSI-R consiste en un cuestionario autoaplicable, con versiones específicas para adolescentes y adultos, que explora el comportamiento en el año previo a la encuesta. Consta de 159 preguntas dirigidas a evaluar la intensidad de los trastornos en diez dominios: problemas de conducta, trastornos de salud, desórdenes afectivos y psicológicos, baja competencia social, disfuncionalidad familiar, baja adherencia escolar, inadecuado desempeño laboral, vinculación con pares desviantes y consumidores de drogas, uso inadecuado del tiempo libre, e intensidad del consumo de bebidas alcohólicas o drogas. Las opciones de respuesta son dicotómicas (presencia o ausencia) y a partir de ellas se pueden estimar índices generales, por dominio y relativos.

La versión del DUSI-R adaptada para adolescentes que se aplicó recababa también datos sociodemográficos y contenía preguntas sobre el consumo de bebidas alcohólicas, tabaco y drogas ilícitas en tres categorías (alguna vez en la vida, en los últimos 12 meses y en los últimos 30 días). Esta versión se adaptó al español usual en México y se cotejó con el original en inglés y otras versiones en español, se sometió a la evaluación de expertos y a una prueba piloto con estudiantes, y posteriormente se hicieron los ajustes pertinentes. Se omitieron las escalas de los dominios "desempeño laboral" (debido a que estudios previos indicaban que solamente una reducida proporción de estudiantes cuenta con empleo y no necesariamente formal y remunerado) y "estado de salud" (ya que explora factores no modificables desde la perspectiva de las intervenciones preventivas institucionales). Se reestructuró el contenido de la escala de disfuncionalidad familiar en su totalidad, debido a la baja confiabilidad 
de la versión original al aplicarse a estudiantes. ${ }^{4}$

De este modo, la versión utilizada quedó conformada por 87 preguntas que - según el análisis realizado inicialmente con la muestra de 1460 estudiantes- registraron parámetros adecuados de confiabilidad y validez (39). El índice general de consistencia interna (alfa de Cronbach) fue de 0,945 , mientras que un análisis con rotación ortogonal de los componentes principales permitió agrupar las preguntas en ocho factores - todos con cargas superiores a $0,4-$ que explicaban $44,2 \%$ de la varianza. Los ocho factores identificados son:

a) Intensidad del consumo de bebidas alcohólicas o drogas: 18 preguntas sobre los síntomas de consumo y dependencia de estas sustancias y problemas de ajuste escolar asociados (alfa de Cronbach $=0,932$ ).

b) Malestar afectivo: 12 preguntas relacionadas principalmente con síntomas de ansiedad y depresión y con una exacerbada sensibilidad afectiva (alfa de Cronbach $=0,852$ ).

c) Bajo control conductual: 14 preguntas sobre conductas problemáticas, el insuficiente control de los impulsos, la agresividad y la irritabilidad (alfa de Cronbach = 0,842).

d) Baja adherencia escolar: 12 preguntas sobre la baja motivación por el estudio, el ausentismo escolar y el bajo rendimiento académico (alfa de Cronbach $=0,857$ ).

e) Baja competencia social: 10 preguntas relacionadas con la baja asertividad, las dificultades de socialización y los sentimientos de rechazo (alfa de Cronbach $=0,821$ ).

f) Relaciones familiares disfuncionales: 9 preguntas vinculadas con los conflictos y la violencia intrafamiliares, el desapego afectivo y el consumo de bebidas alcohólicas y sustancias ilícitas en la familia (alfa de Cronbach $=0,833$ ).

\footnotetext{
4 Rodríguez S, Arellánez J, Díaz B, González D. Ajuste psicosocial y consumo de drogas. México, D.F.: Centros de Integración Juvenil; 1999. (Informe de investigación 97-27).
}

g) Pertenencia a redes sociales desviantes: 8 preguntas relacionadas con la presencia de actitudes y conductas antisociales y el consumo de drogas por amigos del entrevistado (alfa de Cronbach $=0,822$ ).

h) Aislamiento social: 4 preguntas, tres de ellas referidas a los sentimientos de soledad y una al aburrimiento (alfa de Cronbach $=0,755$ ).

A diferencia de la encuesta DUSI-R original, la medición se realizó mediante una escala de tipo Likert, con cinco opciones de frecuencia y un intervalo de medición de la prevalencia y la intensidad de los trastornos de ajuste referido a los últimos 12 meses.

\section{Análisis de los datos}

Las respuestas a la encuesta se introdujeron en una base de datos con un índice de error final menor de $0,2 \%$; se corroboró que los datos faltantes se distribuyeran aleatoriamente y para la imputación de valores se tomó el promedio de la variable correspondiente a partir de toda la muestra (en las variables relacionadas con el consumo de drogas se tomó el promedio correspondiente al grupo de consumidores).

A partir de la hipótesis de que el consumo de drogas ilícitas está asociado con una mayor intensidad de los trastornos de ajuste psicosocial, el análisis de la información consistió, primero, en la realización de la prueba de $t$ de diferencias de medias para muestras independientes entre los estudiantes que habían consumido drogas ilícitas alguna vez en su vida y los que no las habían consumido nunca, tomando en cuenta los índices de intensidad de los ocho dominios de ajuste estudiados, y segundo, en un análisis de correlaciones entre estos índices.

Posteriormente, se llevaron a cabo tres análisis de regresión logística independientes con casos válidos a fin de identificar los factores de predicción del consumo de sustancias ilícitas alguna vez en la vida, en general y por sexo, con sus grados de libertad (gl). La regresión logística se efectuó en dos bloques: en el primero (por el método de "introducir") se emplearon las variables sociodemográficas: sexo, edad, nivel de escolaridad (secundaria o bachillerato) y ocupación (estudia, o estudia y trabaja); en el segundo bloque (por el método "hacia adelante condicional") se incorporaron las variables extraídas de la encuesta DUSI que presentaron valores de $P \leq 0,05$ (40).

Por último, a partir de los resultados de los análisis de correlación y de regresión y con el fin de corroborar la existencia de relaciones de mediación entre los factores estudiados y de identificar variables latentes, se elaboró un modelo de ecuaciones estructurales con los estudiantes consumidores de sustancias ilícitas $(n=254)$ y con las variables de todos los dominios, excepto el de aislamiento social, ya que su inclusión afectaba los estadísticos del modelo. Tanto la prueba como la valoración de las diferentes soluciones en función de los valores de regresión, la varianza explicada, la parsimonia y el ajuste permitieron llegar a un modelo que comprendió, además de los siete factores medidos directamente, dos variables latentes (41).

El análisis de la información se llevó a cabo con los programas SPSS v. $11 \mathrm{y}$ AMOS v. 4.

\section{RESULTADOS}

Al comparar el grupo de consumidores de drogas con el que no las consumía se encontró una proporción significativamente mayor de hombres $\left(59,3 \%\right.$ frente a $50,4 \% ; \chi^{2}=4,066$; $P<0,05)$ y de estudiantes de bachillerato $\left(53,5 \%\right.$ frente a $39,7 \% ; \chi^{2}=9,942$; $P<0,002)$; la edad promedio de los consumidores de sustancias ilícitas fue mayor que la de los que no la consumían $(15,97 \pm 1,51$ años frente a $15,44 \pm 1,24$ años; $t=4,315 ; \mathrm{gl}=489,77$; $P<0,0001)$. Estas diferencias confirman observaciones de otros estudios similares realizados en estudiantes (3). Sin embargo, el modelo de regresión logística desarrollado a fin de controlar el posible efecto de confusión mostró que estas variables carecen de poder de predicción significativo. 
CUADRO 1. Trastornos de ajuste psicosocial en estudiantes que consumieron drogas ilícitas alguna vez en la vida y los que no las consumieron nunca

\begin{tabular}{|c|c|c|c|c|c|c|}
\hline \multirow[b]{2}{*}{ Dominio } & \multicolumn{2}{|c|}{$\begin{array}{l}\text { Consumidores de drogas ilícitas } \\
\qquad(n=254)\end{array}$} & \multicolumn{2}{|c|}{$\begin{array}{l}\text { No consumidores } \\
\quad(n=262)\end{array}$} & \multirow[b]{2}{*}{$\begin{array}{l}\text { Grados de } \\
\text { libertad }\end{array}$} & \multirow[b]{2}{*}{$t$} \\
\hline & Media & $\begin{array}{l}\text { Desviación } \\
\text { estándar }\end{array}$ & Media & $\begin{array}{l}\text { Desviación } \\
\text { estándar }\end{array}$ & & \\
\hline Vínculo con pares desviantes ${ }^{a}$ & 1,81 & 0,80 & 1,05 & 0,72 & 504,17 & $11,203^{b}$ \\
\hline Intensidad del consumo de bebidas & & & & & & \\
\hline alcohólicas y drogas ilícitas ${ }^{a}$ & 0,68 & 0,75 & 0,12 & 0,29 & 325,18 & $11,096^{b}$ \\
\hline Relaciones familiares disfuncionales ${ }^{a}$ & 1,18 & 0,74 & 0,74 & 0,65 & 502,14 & $7,252^{b}$ \\
\hline Malestar afectivo & 1,99 & 0,70 & 1,77 & 0,65 & 514 & $3,802^{b}$ \\
\hline Aislamiento social & 1,73 & 0,94 & 1,46 & 0,90 & 514 & $3,277^{c}$ \\
\hline Baja competencia social & 1,14 & 0,70 & 0,95 & 0,64 & 514 & $3,128^{\circ}$ \\
\hline Índice general de trastornos de ajuste psicosocial & 1,51 & 0,43 & 1,10 & 0,40 & 514 & $11,337^{b}$ \\
\hline
\end{tabular}

\footnotetext{
${ }^{a}$ Los valores de $t y$ los grados de libertad corresponden, de acuerdo con la prueba de Levene para la igualdad de varianzas, al supuesto de existencia de varianzas diferentes en los grupos comparados.

b $P<0,0001$.

c $P=0,001$.

d $P=0,002$.
}

Se detectaron también diferencias significativas entre los estudiantes que consumían drogas ilícitas y los que no las consumían en los ocho dominios estudiados. Los primeros presentaron trastornos de ajuste psicosocial más intensos que los segundos. Como puede observarse en el cuadro 1, las mayores diferencias correspondieron a los trastornos relacionados con la pertenencia a redes sociales desviantes, el propio consumo de sustancias y el bajo control conductual.

Los dominios de malestar afectivo, el bajo control conductual y el aislamiento social presentaron calificaciones relativamente más altas en general, incluso en el grupo de no consumidores, posiblemente como reflejo de las alteraciones propias de la adolescencia. Por el contrario, los trastornos por el consumo de sustancias ilícitas mostraron un índice de intensidad relativamente bajo en ambos grupos, posiblemente porque la mayoría de los consumidores eran experimentales. ${ }^{5}$

Según el análisis de correlación se encontró una asociación moderada

\footnotetext{
5 Los valores de $t$ y los grados de libertad empleados para las relaciones familiares disfuncionales, la pertenencia a redes sociales desviantes y la intensidad del uso de sustancias ilícitas corresponden, de acuerdo con la prueba de Levene para la igualdad de varianzas, al supuesto de varianzas diferentes entre los grupos comparados.
}

$(P<0,005)$ entre la intensidad del consumo de sustancias ilícitas, por una parte, y los problemas de control conductual $(r=0,379)$, la baja adherencia escolar $(r=0,400)$, las relaciones familiares disfuncionales $(r=0,344)$ y la pertenencia a redes sociales desviantes $(r=0,480)$, por la otra. De igual modo, se observó una moderada correlación entre los problemas de control conductual y la baja adherencia escolar $(r=0,462)$ y la pertenencia a redes sociales desviantes $(r=0,448)$. Se encontró una correlación mayor entre el malestar afectivo y la baja competencia social $(r=0,562)$, así como índices intermedios $(r>0,400)$ entre esas dos variables y la disfuncionalidad familiar y el aislamiento social, e igualmente entre el malestar afectivo y la baja adherencia escolar.

Los análisis de regresión logística identificaron cuatro factores de predicción del consumo de drogas ilícitas alguna vez en la vida: el bajo control conductual, la disfuncionalidad familiar, la pertenencia a redes sociales desviantes y la baja adherencia escolar. Al controlar por los posibles factores de confusión (edad, sexo, nivel de escolaridad y ocupación), los factores de predicción del consumo de drogas en la muestra total fueron: estar vinculado con amigos que consumen drogas y presentan actitudes o comportamientos desvian- tes (razón de posibilidades [odds ratio, OR] = 2,393), y un bajo control conductual - es decir, la tendencia a actuar con irritabilidad, agresividad y cediendo a los impulsos- $(\mathrm{OR}=2,152)$. Así mismo, estar expuesto con frecuencia al consumo de sustancias ilícitas y alcohol en el hogar y a una situación familiar conflictiva y violenta, carente de expresiones afectivas, representó un riesgo mayor de usar drogas ilícitas $(\mathrm{OR}=1,876)$. El modelo de regresión explicó 34,3\% de la varianza y clasificó correctamente a 74,3\% de los casos (cuadro 2).

En los varones, se identificaron como factores de predicción del consumo de drogas ilícitas el bajo control conductual $(\mathrm{OR}=2,269)$ y la pertenencia a redes sociales desviantes $(\mathrm{OR}=1,758)$. En este caso, el modelo presentó una menor capacidad explicativa (varianza explicada: 23,6\%), aunque clasificó correctamente 73,9\% de los casos (cuadro 2).

En las mujeres, los factores de predicción del consumo de sustancias ilícitas fueron la exposición a relaciones familiares disfuncionales, la relación con pares que consumían estas sustancias y presentaban actitudes antisociales, y la baja adherencia escolar. Comparada con su ausencia, la presencia de estos factores representó, respectivamente, un riesgo de consumir dro- 
CUADRO 2. Modelos de regresión logística por bloques ${ }^{a}$ para la identificación de factores de predicción del consumo de drogas ilícitas alguna vez en la vida por estudiantes mexicanos

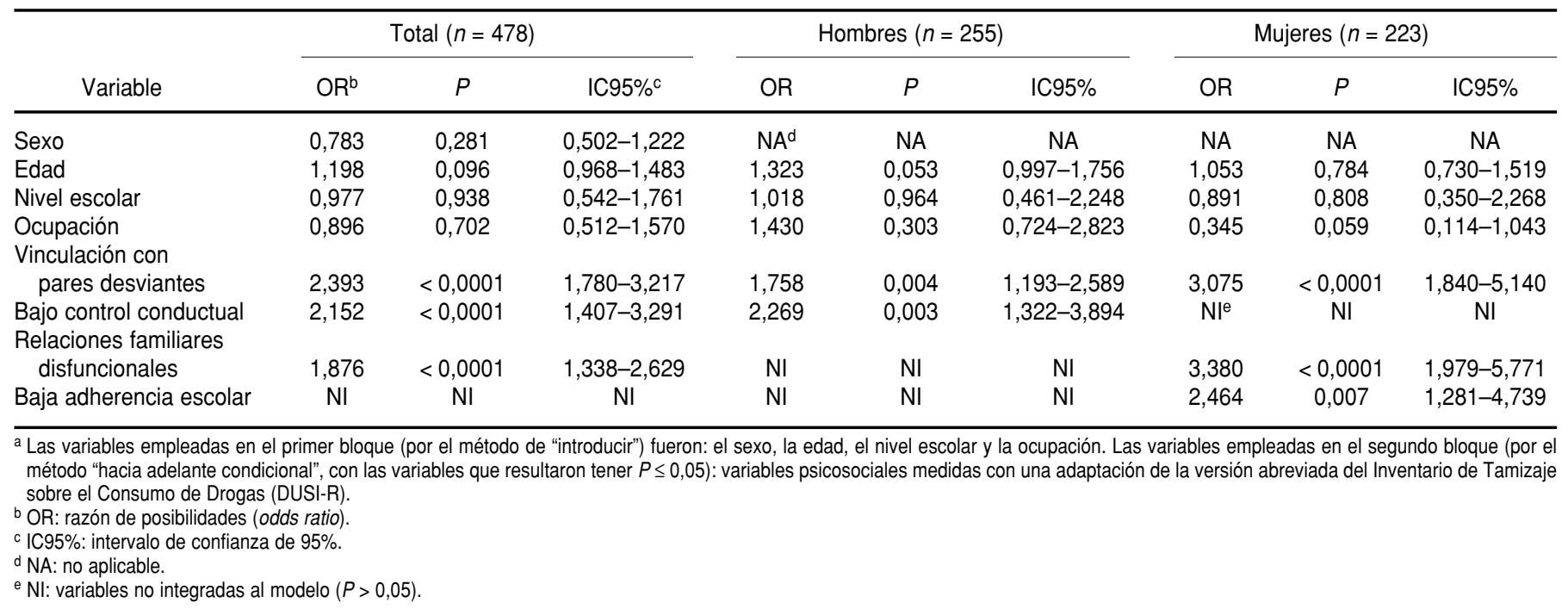

gas $238 \%$, 208\% y $146 \%$ mayor. En este caso, la varianza explicada por el modelo fue de $53,0 \%$, con $63,7 \%$ de casos clasificados correctamente ${ }^{6}$ (cuadro 2).

En cuanto a las posibles relaciones entre los factores estudiados, el análisis realizado permitió llegar a un modelo estructural válido, compuesto por siete factores medidos directamente y dos variables latentes, con indicadores aceptables de parsimonia y ajuste (figura 1). De acuerdo con el valor obtenido de la ji al cuadrado $\left(\chi^{2}=27,198\right.$; $\mathrm{gl}=13 ; P=0,012)$ y el tamaño de la muestra, el modelo puede considerarse aceptable. ${ }^{7} \mathrm{La}$ discrepancia poblacional medida por el error de apro-

6 La estimación de OR tendió a ser más precisa en el modelo general, en particular en el caso del factor "relaciones familiares disfuncionales". En el modelo de los varones, la estimación más precisa correspondió a la pertenencia a redes sociales desviantes, mientras que en mujeres, en general, las estimaciones fueron menos precisas, por lo que deben tomarse con más cautela.

7 El tamaño de la muestra suele influir en el valor de la ji al cuadrado y su nivel de significación. Cuando la muestra es grande, como en este caso, un nivel estadísticamente significativo no implica necesariamente un inadecuado ajuste del modelo. Para controlar el efecto del tamaño de la muestra, Kline (41) recomienda dividir el valor de la ji al cuadrado entre los grados de libertad y considera aceptables los modelos con una razón menor de 3 . En este caso, la razón fue de 2,09, por lo que el modelo se consideró aceptable. ximación cuadrático medio fue de 0,066 (IC90\%: 0,03-0,10) lo que, igualmente, indica un ajuste satisfactorio del modelo en relación con los grados de libertad. También el índice de bondad de ajuste se situó en un nivel aceptable de 0,973 .

Según el modelo resultante, la intensidad de consumo de bebidas alcohólicas o drogas ilícitas puede formar parte de un factor latente "trastornos de ajuste conductual", junto con la dificultad para integrarse al medio escolar y la conducta impulsiva y agresiva.

FIGURA 1. Modelo estructural de los factores psicosociales asociados con el consumo de bebidas alcohólicas y drogas en estudiantes mexicanos, con los valores de regresión estandarizados $^{\mathrm{a}}$

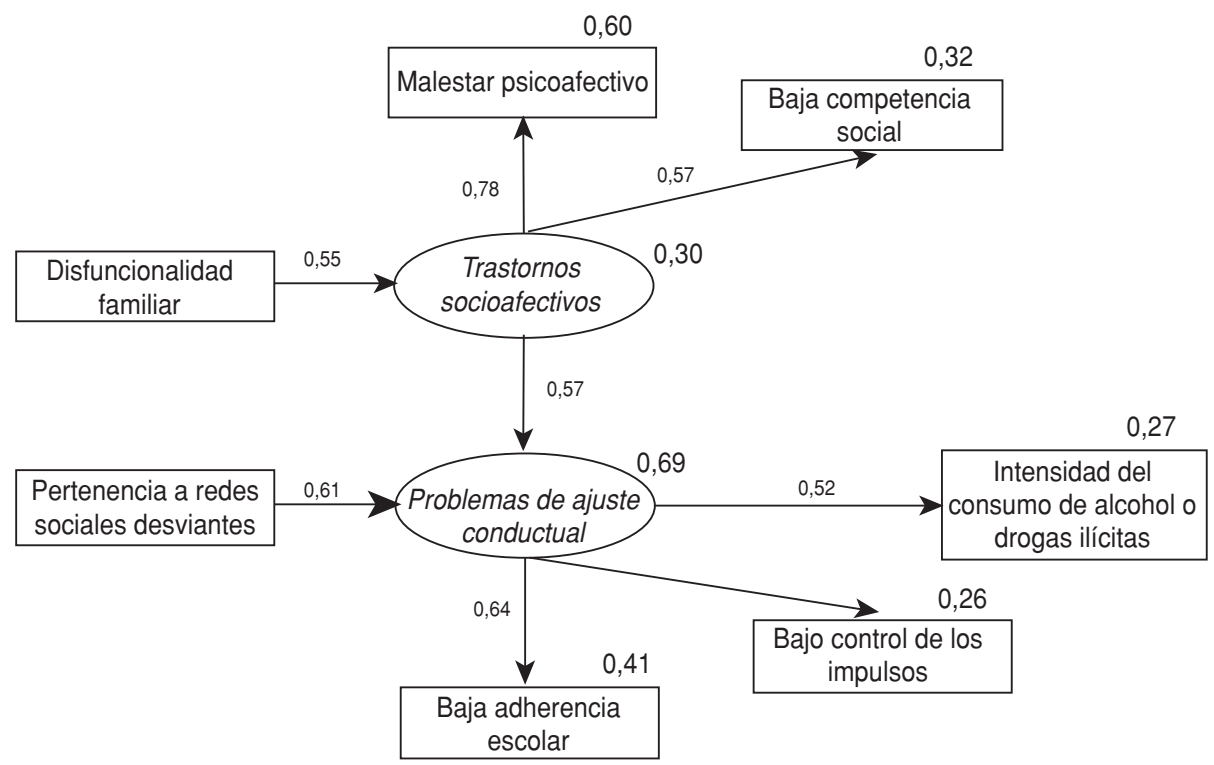

a En cifras pequeñas se muestran los coeficientes de regresión estandarizados; en cifras mayores, la proporción de la varianza explicada de cada factor. 
Este factor explicaría tanto la intensidad del consumo de bebidas alcohólicas y sustancias ilícitas como la baja adherencia escolar y el bajo control conductual y de impulsos, con índices de regresión estandarizados superiores a 0,5 y varianzas explicadas de $27 \%, 41 \%$ y $26 \%$, respectivamente. A su vez, esta variable estaría determinada por la vinculación con pares desviantes y por un factor latente adicional, denominado "trastornos socioafectivos", los cuales en conjunto explicarían $69 \%$ de la varianza, con índices estandarizados de regresión de 0,61 y 0,57 , respectivamente.

El factor "trastornos socioafectivos" agrupó a las variables "malestar psicoafectivo" y "baja competencia social", y explicó $60 \%(\beta=0,78)$ y $32 \%$ $(\beta=0,57)$ de sus respectivas varianzas. Este factor indicaría la presencia de dificultades de socialización y falta de habilidades de comunicación asertiva asociadas con un estado de depresión. La pertenencia a un grupo familiar disfuncional, conflictivo y violento, carente de expresiones de afecto y en el que se consumen sustancias ilícitas puede, a su vez, explicar $30 \%$ de la varianza del factor latente "trastornos socioafectivos" $(\beta=0,55)$.

\section{DISCUSIÓN}

En primera instancia, los hallazgos expuestos reflejan claramente la existencia en la muestra de diferencias significativas en términos de ajuste psicosocial entre los estudiantes que habían consumido drogas ilícitas y los que no las habían consumido. Así mismo, indican que se puede explicar el consumo de estas drogas - al menos en su fase experimental, es decir, alguna vez en la vida-, primero, por factores interpersonales como la exposición a condiciones familiares de conflicto, violencia, falta de apoyo afectivo y consumo de bebidas alcohólicas y de sustancias ilícitas, y como el vínculo con pares que consumen estas sustancias y mantienen conductas o actitudes desviantes; y segundo, por factores individuales de vulnerabilidad, como el malestar afectivo y la tendencia a la irritabilidad, la agresividad y la falta de control de los impulsos. Las diferencias observadas entre los estudiantes y las estudiantes de la muestra pueden deberse a que el consumo de drogas en las mujeres se relaciona más claramente con factores de índole interpersonal (concretamente, la disfuncionalidad familiar y una baja adherencia escolar) que en los varones, en los que es mayor el peso de factores de predicción individual, como el bajo control conductual.

De manera más específica, el modelo de ecuaciones estructurales desarrollado demostró que el consumo de drogas puede situarse - al menos para esta muestra- como un elemento de un complejo cuadro de trastornos de ajuste. Entre los elementos de este cuadro se encuentran las dificultades para la integración social, con una tendencia a adoptar conductas impulsivas o agresivas y dificultades para responder adecuadamente a las demandas de ajuste del medio escolar. Este cuadro se vería agravado por la interacción con amigos desviantes y los antecedentes de trastornos socioafectivos, vinculados a su vez, por un lado, con situaciones de malestar afectivo (depresión, ansiedad, hipersensibilidad) $\mathrm{y}$, por otro, con dificultades para la integración social, sentimientos de rechazo y la falta de habilidades de comunicación asertiva. Finalmente, la intensidad de estos problemas "socioafectivos" parece estar determinada por la pertenencia a un contexto familiar disfuncional, conflictivo $y$ distante.

De esta manera, el modelo planteado muestra cómo los trastornos de ajuste conductual, a los que se incorpora el consumo de drogas ilícitas, pueden explicarse de manera directa por la influencia desviante del grupo de pares, conjugada con la inhabilidad para enfrentarla asertivamente y con los antecedentes de malestar afectivo y de dificultades para la integración social. Por su parte, la disfuncionalidad familiar tendría una influencia indirecta y mediada sobre el consumo de sustancias ilícitas y los problemas de conducta, al influir en la aparición de síntomas de malestar psicoafectivo y en un insuficiente desarrollo de habilidades comunicativas y sociales.

Tomados en conjunto, estos hallazgos ponen de manifiesto la interacción de factores individuales y psicosociales, y concuerdan con lo sostenido por Calafat y colaboradores (42) en el sentido de que, desde el punto de vista del individuo, el consumo de sustancias ilícitas está relacionado con la expresión externalizada del conflicto psíquico (tendencia a actos impulsivos violentos y agresivos con bajo control, conocidos como acting out), al tiempo que desde una perspectiva psicosocial se asocia con la presencia de actitudes y conductas que dificultan la integración a la sociedad convencional.

Estos resultados confirman, en lo general, otros estudios realizados con el DUSI-R en México y América Latina, en los que los trastornos de control conductual, los problemas familiares y la relación con pares que presentan conductas desviantes se identificaron como factores asociados significativamente con el consumo de drogas ilícitas (32-38). ${ }^{8}$

Sin embargo, se presentan también algunos aspectos novedosos. En primer lugar, la adaptación del DUSI-R utilizada cuenta con índices más altos de consistencia interna general y por dominios, lo que proporcionó una base más sólida para la prueba de hipótesis; además, la utilización de una escala ajustada de "relaciones familiares disfuncionales" que incorporó indicadores de violencia familiar, permitió identificar factores de predicción significativos en una esfera de ajuste psicosocial que el DUSI no había identificado hasta ahora. En segundo lugar, el análisis de los factores de predicción por sexo permitió demostrar que la relación entre el consumo de drogas ilícitas y la baja adherencia escolar fue significativa únicamente en las mujeres, algo no encontrado en otros estudios realizados en México con muestras similares de estudiantes. Por último, un importante aporte fue identificar las relaciones de predicción y mediación existente entre los facto-

\footnotetext{
8 Ver, también, las referencias señaladas en la nota 3.
} 
res analizados y las variables latentes. Así, este estudio contribuye al desarrollo de modelos explicativos y abre el camino para diseñar programas y acciones para enfrentar el grave problema del consumo de drogas ilícitas por adolescentes, con una clara distinción entre los factores próximos y distantes.

De esta manera, el presente estudio aportó elementos empíricos que podrían explicarse mediante modelos teóricos ya probados sobre el consumo experimental de sustancias ilícitas en adolescentes (7-9). La relación observada entre los factores de consumo de esas sustancias en el seno de la familia y por sus pares corrobora los planteamientos de las teorías del aprendizaje social con respecto al modelamiento del consumo de drogas y las actitudes que lo favorecen, así como al efecto del reforzamiento social, aunado a una baja competencia para enfrentar la influencia de los otros. De modo similar, las teorías relativas a la desvinculación de las instancias sociales convencionales, así como otros modelos psicológicosociales (9), podrían tomarse como referentes para explicar cómo las carencias psicosociales en el seno familiar dificultan el desarrollo de habilidades de socialización y suscitan alteraciones psicoafectivas que más tarde -en interacción con la influencia de pares desviantes - repercuten en el debilitamiento de la motivación para adherirse a las normas convencionales (en este caso, reflejada en una baja adherencia escolar) y en la propensión a consumir sustancias ilícitas.

Estos resultados y los posibles marcos explicativos destacan la pertinencia de diseñar y poner en práctica acciones y estrategias preventivas que aborden este problema - principallas Adicciones. El consumo de drogas en México. México, D.F.: Secretaría de Salud; 1999. mente en grupos de alto riesgo como los adolescentes escolarizados- mediante el desarrollo de habilidades para el control de impulsos, así como para afrontar las relaciones familiares problemáticas y responder adecuadamente a las demandas de ajuste del medio escolar. Con ello se podrían fomentar mejores condiciones de integración social y una mayor adherencia escolar, con el consecuente efecto de reforzamiento y retroalimentación positivos, la disminución del fracaso escolar y el aumento del apego a las normas sociales. Con respecto a la influencia de los pares, se confirmó la importancia de promover el desarrollo de habilidades de comunicación asertiva para enfrentar la presión social, pero también de fortalecer la competencia social y las habilidades de relación interpersonal para reforzar la autoeficacia y la integración a grupos de jóvenes no consumidores, mejor integrados al medio social convencional.

Por otro lado, se debe fomentar el consumo moderado de sustancias lícitas en el hogar y la abstinencia de consumir las ilícitas, promover prácticas adecuadas de tutoría y supervisión parental, desarrollar pautas relacionales nutricias y de apoyo afectivo en la familia y promover recursos para la prevención de la violencia intrafamiliar. De acuerdo con el modelo estructural propuesto, estas medidas podrían contribuir a reducir la incidencia de trastornos psicoafectivos entre los jóvenes y fortalecer su autoestima y capacidad de integración social.

Este estudio presentó limitaciones que deben tomarse en cuenta, entre ellas la utilización de una muestra no probabilística, el levantamiento transversal de los datos y la aplicación de un esquema limitado de medición de

\section{REFERENCIAS}

2. Secretaría de Salud, Instituto Nacional de Estadística, Geografía e Informática. Encuesta Nacional de Adicciones 2002. México, D.F.: Instituto Nacional de Estadística, Geografía e In- variables. Por ello se subraya la conveniencia de corroborar los resultados obtenidos mediante estudios prospectivos, que permitan identificar los factores de vulnerabilidad y de riesgo propiamente dichos y no solo factores de predicción basados en cálculos probabilísticos no experimentales.

Estudios futuros podrían emplear muestras probabilísticas de poblaciones específicas —además de muestras representativas de los estudiantes de educación media- para contar con parámetros generalizables. También podrían hacerse mediciones desagregadas de los factores que en el presente estudio fueron agrupados en dominios de ajuste genéricos y que merecerían una atención especial para discriminar mejor su aportación al consumo de sustancias ilícitas. Entre estos factores se encuentran el desapego familiar, la falta de supervisión paterna y de pautas claras de autoridad, el consumo de drogas ilícitas por personas cercanas significativas, la tendencia a actuar impulsivamente diferenciada de la conducta agresiva o violenta, la baja tolerancia a la frustración, los trastornos depresivos y de ansiedad, la falta de habilidades asertivas o de recursos para la interacción y el déficit de habilidades particulares o la falta de motivación para el adecuado desempeño escolar. De igual modo, sería recomendable ahondar en el estudio de las diferencias de género observadas en este estudio, probar los modelos predictivos elaborados con otros grupos poblacionales (por ejemplo, jóvenes de mayor edad y menores de la calle) y ampliar la investigación a factores de predicción de otras conductas, además del consumo experimental de drogas ilícitas, como la transición al consumo habitual y la dependencia. formática; 2004. Hallado en http:/ / www. inegi. gob.mx/prod_serv/contenidos/espanol/ bvinegi/productos/continuas/sociales/salud/ 2004/Ena02.pdf. Acceso el 20 de mayo de 2008. 
3. Villatoro J, Hernández I, Hernández H, Fleiz C, Blanco J, Medina-Mora ME. Encuesta en la Ciudad de México sobre el uso de drogas entre la comunidad escolar 2003. Encuestas de Consumo de Drogas de Estudiantes III, 1991-2003. México, D.F.: SEP-INPRFM; 2004. Hallado en http://www.inprf.org.mx/avisos/ encuestas/Encuesta_SS.htm. Acceso el $20 \mathrm{de}$ mayo de 2008.

4. Secretaría de Salud. SISVEA, Sistema de Vigilancia Epidemiológica de las Adicciones. México, D.F.: Secretaría de Salud; 2002. Hallado en http://www.dgepi.salud.gob.mx/ sis/descrip.htm. Acceso el 20 de mayo de 2008.

5. Gutiérrez A, Castillo I. Consumo de drogas en pacientes de primer ingreso a tratamiento en centros de integración juvenil, enero-junio 2006 [sitio en Internet]. México, D.F.: Centros de Integración Juvenil. Disponible en: http://www.cij.gob.mx/Paginas/PDF/ Especialistas/0701.pdf. Acceso el 5 de julio de 2008.

6. Gardner S, Brounstein P, Stone D. Sciencebased substance abuse prevention: a guide. Rockville, Maryland: Substance Abuse and Medical Health Service Administration, Center for Substance Abuse Prevention; 2001.

7. Becoña E. Bases teóricas que sustentan los programas de prevención de drogas. Madrid: Ministerio del Interior, Delegación del Gobierno para el Plan Nacional sobre Drogas; 1999.

8. Becoña E. Bases científicas de la prevención de las drogodependencias. Madrid: Ministerio del Interior, Delegación del Gobierno para el Plan Nacional sobre Drogas; 2002.

9. Petraitis J, Flay B, Miller T. Reviewing theories of adolescent substance use: organizing pieces in the puzzle. Psychol Bull. 1995; 117(1):67-86.

10. Hawkins DJ, Lishner D, Catalano R. Childhood predictors and the prevention of adolescent substance abuse. En: Jones C, Battjes R, eds. Etiology of drug abuse: implications for prevention. Rockville, Maryland: National Institute on Drug Abuse; 1985. Pp. 75-126. (NIDA Res Monogr Ser No. 56.)

11. Hawkins DJ, Catalano R, Miller J. Risk and protective factors for alcohol and other drug problems in adolescence and early adulthood: implications for substance abuse prevention. Psychol Bull. 1992;112(1):64-105.

12. Guo J, Hawkins DJ, Hill K, Abbott R. Childhood and adolescent predictors of alcohol abuse and dependence in young adulthood. J Stud Alcohol. 2001;62(6):754-62.

13. Arthur M, Hawkins DJ, Pollard J, Catalano R, Baglioni A. Measuring risk and protective factors for substance use, delinquency, and other adolescent problem behaviors. Eval Rev. 2002;26(6):575-601.

14. Newcomb M. Identifying high-risk youth: prevalence and patterns of adolescent drug abuse. En: Rahdert E, Czechowicz D, eds. Adolescent drug abuse: clinical assessment and therapeutic interventions. Rockville, Maryland: National Institute on Drug Abuse; 1995. Pp. 7-37. (NIDA Res Monogr Ser No. 156.)
15. Murray D, Perry C. The prevention of adolescent drug abuse: implications of etiological, developmental, behavioral, and environmental models. En: Jones C, Battjes R, eds. Etiology of drug abuse: implications for prevention. Rockville, Maryland: National Institute on Drug Abuse; 1985. Pp. 236-56. (NIDA Res Monogr Ser No. 56.)

16. Spooner C, Mattick R, Noffs W. A study of the patterns and correlates of substance use among adolescents applying for drug treatment. Aust N Z J Public Health. 2000;24(5): 492-502.

17. Ripple C, Luthar S. Familial factors in illicit drug abuse: an interdisciplinary perspective. Am J Drug Alcohol Abuse. 1996;22(2):147-72.

18. Medina-Mora ME, Villatoro J, López E, Berenzon S, Carreño S, Juárez F. Los factores que se relacionan con el inicio, el uso continuado y el abuso de sustancias psicoactivas en adolescentes mexicanos. Gac Med Mex. 1995;131:383-7.

19. Medina-Mora ME, Cravioto P, Villatoro J, Fleiz C, Galván F, Tapia R. Consumo de drogas entre adolescentes: resultados de la Encuesta Nacional de Adicciones, 1998. Salud Publica Mex. 2003;45 (supl1):16-25.

20. Arellánez J, Díaz B, Wagner F, Pérez V. Factores psicosociales asociados con el abuso y la dependencia de drogas entre adolescentes: análisis bivariados de un estudio de casos y controles. Salud Mental. 2004;27(3):54-64.

21. Nazar A, Tapia R, Villa A, León G, MedinaMora ME, Salvatierra B. Factores asociados al consumo de drogas en adolescentes de áreas urbanas de México. Salud Publica Mex. 1994;36(6):646-54.

22. Villatoro J, Medina-Mora ME, Juárez F, Rojas E, Carreño S, Berenzon S. Drug use pathways among high school students of Mexico. Addiction. 1998;93(10):1577-88.

23. Castro ME. Factores de protección asociados al riesgo del consumo de sustancias adictivas en población de jóvenes estudiantes. En: Tapia R, coord. Las adicciones: dimensión, impacto y perspectivas. $2 .^{a}$ ed. México, D.F.: Manual Moderno; 2001. Pp. 277-89.

24. Rodríguez S, Pérez V. Resiliencia y consumo de drogas entre estudiantes de secundaria. Psicol Iberoam. 2002;10(2):42-7.

25. Gracia S, Saldívar A, Contreras C. Validación de la escala de búsqueda de sensaciones: rasgo de personalidad y su importancia en la adicción a las drogas. Psicol Social Mex. 2002;IX:411-8.

26. Tarter R, Laird S, Kabene M, Bukstein O, Kaminer Y. Drug abuse severity in adolescents is associated with magnitude of deviation in temperament traits. $\mathrm{Br} \mathrm{J}$ Addict. 1990;85(11):1501-4.

27. Mezzich A, Tarter R, Kirisci L, Clark D, Bukstein O, Martin C. Subtypes of early age onset alcoholism. Alcohol Clin Exp Res. 1993;17(4): 767-70.

28. Mezzich A, Tarter R, Kirisci L, Hsieh Y, Grimm M. Coping capacity in female adolescent substance abusers. Addict Behav. 1995; 20(2):181-7.

29. Giancola P, Mezzich A. Executive functioning, temperament, and drug use involvement in adolescent females with a substance use disorder. J Child Psychol Psychiatry. 2003; 44(6):857-66.

30. Kirisci L, Mezzich A, Tarter R. Norms and sensitivity of the adolescent version of the Drug Use Screening Inventory. Addict Behav. 1997;20(2):149-57.

31. Tarter R, Kirisci L, Mezzich A. Multivariate typology of adolescents with alcohol use disorder. Am J Addict. 1997;6(2):150-8.

32. Moss H, Bonicatto S, Kirisci L, Girardelli A, Murrelle L. Substance abuse and associated psychosocial problems among Argentina adolescents: sex heterogeneity and familial transmission. Drug Alcohol Depend. 1998;52(3): 221-30.

33. De Micheli D, Formigoni M. Screening of drug use in a teenage Brazilian sample using the Drug Use Screening Inventory. Addict Behav. 2000;25(5):683-91.

34. Sandi L, Díaz A, Ugalde F. Drug use and associated factors among rural adolescents in Costa Rica. Subst Use Misuse. 2002;37(5-7): 599-611.

35. Vittetoe K, López M, Delva J, Wagner F, Anthony J. Behavioral problems and tobacco use among adolescents in Central America and the Dominican Republic. Rev Panam Salud Publica. 2002;11(2):76-82.

36. Gosebruch G, Sánchez M, Delva J, Wagner F, Anthony J. Family attention and tobacco smoking among adolescents in Central America, Panama, and the Dominican Republic. Subst Use Misuse. 2003;38(8):1037-62.

37. De Micheli D, Formigoni M. Drug use by Brazilian students: associations with family, psychosocial, health, demographic and behavioral characteristics. Addiction. 2004; 99(5): 570-8.

38. Díaz B, Arellánez J, Martínez J. Uso de drogas $\mathrm{y}$ factores psicosociales asociados entre estudiantes de educación media básica del estado de Nuevo León. En: Medina-Mora ME, coord. Observatorio mexicano en tabaco, alcohol y otras drogas, 2002. México, D.F.: Consejo Nacional contra las Adicciones; 2002. Pp. 133-6.

39. Díaz B, González D, García R. Adaptación del Drug Use Screening Inventory para su aplicación con adolescentes mexicanos. Adicciones. 2006;18(2):197-210.

40. Pardo A, Ruiz M. SPSS II, guía para el análisis de datos. México, D.F.: McGraw Hill; 2002.

41. Kline R. Principles and practice of structural equation modeling. New York: Guildford Press; 1998.

42. Calafat A, Amengual M, Palmer A, Sabila C. Drug use and its relationship to other behavior disorders and maladjustment signs among adolescents. Subst Use Misuse. 1997;32(1):1-24.

Manuscrito recibido el 16 de mayo de 2007. Aceptado para publicación, tras revisión, el 3 de mayo de 2008. 
ABSTRACT Objectives. To identify psychosocial risk factors for substance abuse among Mexican students and to offer elements for the design of prevention programs.

\section{Psychosocial risk factors for illicit drug use in a sample of Mexican high school students}

Key words Street drugs, risk factors, students, Mexico.

\section{Key words . Street drugs, risk factors, students, Mexico.}

Methods. A cross-sectional, nonexperimental study of a sample of 516 high school students in six of Mexico's most important cities. From April-June 2005, a customized version of the Drug Use Screening Inventory (revised) (DUSI-R) was administered. The analysis comprised eight factors: alcohol and drug abuse, affective disorders, poor self-control, poor school adjustment, low social competence, dysfunctional family relationships, social isolation, and being part of a detrimental social network (whose members take drugs and have antisocial attitudes). Factors predictive for illicit drug use were found by logistical regression, and a structural equation model was designed to determine the relationships among the factors.

Results. The factors that predicted substance abuse were poor self-control with a tendency to act impulsively and aggressively; associating with troublemakers; and being frequently exposed to family conflicts, violence, and drug and/or alcohol use in the home. The structural equation model indicated that substance abuse is one of a group of disorders directly determined by associating with detrimental peers, and a higher rate of socioaffective disorders, and indirectly, by dysfunctional family relationships.

Conclusions. Some of the suggestions made by theoretical models to explain substance abuse were confirmed. These empirically-supported elements can contribute to the design of prevention programs, especially those that are selective and recommended.

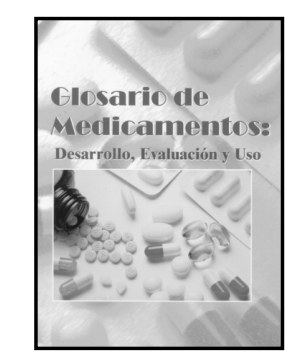

$1999 \cdot 312 \mathrm{pp}$.

ISBN 9275323054 Código: OT $118 \cdot$

Precio: US\$ 18.00 en

América Latina y el

Caribe/ US\$20.00 en el resto del mundo

\section{Glosario de medicamentos: desarrollo, evaluación y uso}

Este libro no es solo una simple descripción de términos. Con el fin de facilitar al lector el uso de la literatura especializada en inglés, en él se ha incluido el equivalente, en ese idioma, de cada uno de los términos. El libro contiene, además, algunas advertencias en cuanto a las traducciones del inglés al español de términos relacionados con medicamentos, una hoja de instrucciones, una lista de abreviaturas, acrónimos, siglas, símbolos y sinónimos, así como un índice en inglés de los términos incluidos en el glosario.

Este glosario es de gran utilidad para los profesionales de la farmacología, de la medicina y de carreras afines que de una manera $u$ otra se encuentran vinculados con organismos oficiales encargados de evaluar, registrar, seleccionar y velar por el control de calidad de los medicamentos, así como de investigar su uso. 